Journal of Computational Acoustics, Vol. 25, No. 4 (2017) 1650020 (20 pages)

(C) The Author(s)

DOI: $10.1142 / S 0218396 X 1650020 X$

\title{
Infinite Elements and Their Influence on Normal and Radiation Modes in Exterior Acoustics
}

\author{
Lennart Moheit* and Steffen Marburg \\ Chair of Vibroacoustics of Vehicles and Machines \\ Department of Mechanical Engineering \\ Technische Universität München \\ Boltzmannstraße 15, 85748 Garching, Germany \\ *Lennart.Moheit@tum.de
}

Received 9 May 2016

Accepted 14 July 2016

Published 14 March 2017

\begin{abstract}
Acoustic radiation modes (ARMs) and normal modes (NMs) are calculated at the surface of a fluid-filled domain around a solid structure and inside the domain, respectively. In order to compute the exterior acoustic problem and modes, both the finite element method (FEM) and the infinite element method (IFEM) are applied. More accurate results can be obtained by using finer meshes in the FEM or higher-order radial interpolation polynomials in the IFEM, which causes additional degrees of freedom (DOF). As such, more computational cost is required. For this reason, knowledge about convergence behavior of the modes for different mesh cases is desirable, and is the aim of this paper. It is shown that the acoustic impedance matrix for the calculation of the radiation modes can be also constructed from the system matrices of finite and infinite elements instead of boundary element matrices, as is usually done. Grouping behavior of the eigenvalues of the radiation modes can be observed. Finally, both kinds of modes in exterior acoustics are compared in the example of the cross-section of a recorder in air. When the number of DOF is increased by using higher-order radial interpolation polynomials, different eigenvalue convergences can be observed for interpolation polynomials of even and odd order.
\end{abstract}

Keywords: Infinite element method (IFEM); normal modes; acoustic radiation modes.

\section{Introduction}

The discretization and calculation of an acoustic exterior problem involves the problem of an infinite, unbounded domain with a nonreflecting boundary condition at the outside of the fluid-filled domain. Finite elements are only applicable for the discretization

This is an Open Access article published by World Scientific Publishing Company. It is distributed under the terms of the Creative Commons Attribution 4.0 (CC-BY) License. Further distribution of this work is permitted, provided the original work is properly cited. 


\section{Moheit \&3 S. Marburg}

of inner, circumscribed domains and imply reflecting outer borders. Two approaches have been established in order to calculate acoustic exterior problems numerically: Perfectly matched layers (PML) ${ }^{1}$ and the infinite element method (IFEM). ${ }^{2,3}$ The method of conjugated Astley-Leis infinite elements is applied by the authors, since these elements provide the frequency-independent system matrices of stiffness, damping and mass on the basis of the corresponding FE matrices. These system matrices are required for further investigations in modal decomposition. The IFEM implies the Sommerfeld radiation condition, ${ }^{2,4}$ which provides a nonreflective boundary condition and decay to zero of the sound pressure at infinity. The sound pressure field in the radial direction in the domain with the infinite elements is interpolated by polynomials such as Lagrange polynomials, Legendre polynomials or Jacobi polynomials, which lead to differences in the matrix condition number of the discrete, global system matrices. ${ }^{5,6}$

The subject of this paper is the investigation of the influence of the choice of finite elements and the polynomial type for infinite element interpolation in the radial direction as well as the choice of the order of these polynomials on modes in exterior acoustics. The authors consider acoustic radiation modes (ARMs) ${ }^{7-9}$ and normal modes (NMs) ${ }^{10-12}$ which are based on eigenvalue problems of the acoustic impedance matrix $\mathbf{Z}_{R}$ and of a statespace formulation consisting of the discrete system matrices of stiffness, damping and mass, respectively.

The concept of radiation modes can be traced back to investigations of radiating velocity distributions carried out by Borgiotti ${ }^{7}$ and Photiadis ${ }^{8}$ and eigenvalue analysis of the real part of the impedance matrix performed by Sarkissian. ${ }^{9}$ Cunefare and Currey ${ }^{13-15}$ focus on the grouping behavior of the eigenvalues and radiation efficiencies. This line of research is further explored by Peters et $a .^{16}$ who investigate the symmetry characteristics of the real impedance matrix. Recent studies by $\mathrm{Wu}$ et $a{ }^{17}{ }^{17}$ investigate fast multipole BEM and iterative methods for efficiently and accurately calculating the ARMs and the radiation efficiencies in the example of a baffled plate. Marburg et al. ${ }^{18}$ apply ARMs in order to obtain surface contributions to the radiated sound power, which they compare to the acoustic intensity. Liu and Maury ${ }^{19}$ focus on improvements to the ARMs in the near field of vibrating structures.

ARMs are regarded as frequency-dependent modes, because they have to be calculated separately for each frequency as opposed to NMs, which are obtained by solving of a single frequency-independent eigenvalue problem. The concept of NMs is extensively presented by Marburg et al. ${ }^{10-12}$ Investigations of search algorithms for NM eigenvalues were carried out by Fuß et $a .^{20}$ with the example of a recorder as a long, slender and hollow object. By solving the Galbrun equation with an application of infinite elements, Retka and Marburg ${ }^{21}$ solve the state-space eigenvalue problem for a duct with a thin wall and openings on both sides, while taking flow into consideration.

In the context of this paper, the authors will calculate the acoustic impedance matrix $\mathbf{Z}_{R}$ from FEM and IFEM system matrices rather than the boundary element method (BEM) as is suggested in the literature. ${ }^{16,22}$ Recent studies on BEM in exterior acoustics were 
published by Ramesh et al. ${ }^{23}$ and Marburg. ${ }^{24,25}$ The method is also applied for eigenvalue analysis in the exterior acoustic problem by Zheng et al. ${ }^{26}$

The convergence of the infinite elements was shown by Demkowicz and Gerdes ${ }^{27,28}$ and proves the reliability of the approach, but there have been no studies yet on the influence of the infinite elements on modes in exterior acoustics. This paper will first give an overview of the formulation of the infinite elements and of the suitability of the considered polynomials as radial interpolation functions. Following this, both modes in exterior acoustics are summarized. In the simple example model of a circle in an unbounded domain, the reliability of the ARM approach is validated, using FEM and IFEM instead of BEM. Subsequently, the model of a cross-section of a recorder is considered.

Both ARMs and NMs are compared to each other and the influence of different radial interpolation polynomials and of different FE mesh sizes on the modes is discussed and interpreted. Afterwards, their convergence behavior is investigated as the degree of the radial interpolation polynomial is increased. Here, the convergence of the NM eigenvalues is obtained by application of the Modal Assurance Criterion (MAC). ${ }^{29}$ Two different convergences are observed for even and odd orders of the interpolation polynomials for both ARM and NM eigenvalues.

\section{Infinite Element Formulation}

\subsection{Acoustics and discretization of the unbounded domain}

The spatial sound pressure field $p(\mathbf{x})$ in a fluid-filled domain can be described by the Helmholtz equation

$$
\nabla^{2} p(\mathbf{x})+k^{2} p(\mathbf{x})=0, \quad \mathbf{x} \in \Omega \subset \mathbb{R}^{2},
$$

where $k=\omega / c_{f}$ is the wave number with the angular frequency $\omega=2 \pi f$ and the speed of sound in the fluid $c_{f}$. At infinity the nonreflective Sommerfeld radiation condition causes the sound pressure to decay to zero. ${ }^{2,4}$ On the surface of an obstacle, the normal fluid particle velocity $v_{f}(\mathbf{x})$ is assumed to be equal to the structural particle velocity $v_{s}(\mathbf{x})$. In other words, the coupling of the fluid and the structure is nondispersive.

Applying the finite element method (FEM) and IFEM leads to a discrete system of linear equations ${ }^{30}$

$$
\left(\mathbf{K}-i k \mathbf{D}-k^{2} \mathbf{M}\right) \mathbf{p}=i \omega \rho_{f} \boldsymbol{\Theta} \mathbf{v}_{s}=\mathbf{f},
$$

where $\rho_{f}$ is the density of the fluid, $\mathbf{K}, \mathbf{D}$ and $\mathbf{M}$ are the system matrices for stiffness, damping and mass and $\Theta$ is the boundary mass matrix, which was given by Marburg and Nolte ${ }^{30}$ in discrete form

$$
\boldsymbol{\Theta}=\int_{\Gamma} \Phi(\mathbf{x}) \Phi^{T}(\mathbf{x}) \mathrm{d} \Gamma(\mathbf{x}) .
$$


The basis functions $\Phi(\mathbf{x})$ are required for the discretization of the sound pressure and the structural velocity at the surface of a solid structure in the fluid-filled domain

$$
\begin{gathered}
p(\mathbf{x})=\sum_{l=1}^{N} \phi_{l}(\mathbf{x}) p_{l}=\Phi^{T}(\mathbf{x}) \mathbf{p} \\
v_{s}(\mathbf{x})=\sum_{l=1}^{N} \phi_{l}(\mathbf{x}) v_{s, l}=\Phi^{T}(\mathbf{x}) \mathbf{v}_{s} .
\end{gathered}
$$

In the finite element domain, typical linear or quadratic Lagrangian basis functions as described by Ihlenburg ${ }^{4}$ are applied.

The subsequent considerations use the concept of the so-called (mapped and conjugated) Astley-Leis infinite elements in reference to works by Astley et al. , $^{2,3,31,32}$ as well as by Shirron and Babuška. ${ }^{5}$ These elements are attached to the outer boundary of the finite elements to extend the fluid to infinity in the radial direction. The coordinate mapping is given in detail by Marques and Owen. ${ }^{33}$ Here, it is sufficient to state that the global radial coordinate $r$ is related to the local radial coordinate $s$ by

$$
\frac{a(t)}{r}=\frac{1-s}{2}
$$

where $a(t)$ is the radius of the circular boundary line between the FE and IFE domains as a function of the elementwise, local transverse coordinate $t$. Both $s$ and $t$ are defined in the range $[-1,1]$. In the mapping in Eq. (6), the local radial distances $s=-1, s=0$ and $s=1$ correspond to $r=a(t), r=2 a(t)$ and $r=\infty$ in the global space, respectively.

The frequency-dependent basis functions are constructed using an interpolation function $I_{l}(s, t)$ and a phase function $\mu(s, t)$, both of which are functions of the mapped coordinates $s$ and $t$

$$
\phi_{l}(s, t, \omega)=I_{l}(s, t) e^{-i k \mu(s, t)},
$$

where the phase function is given by

$$
\mu(s, t)=a(t) \frac{1+s}{1-s} .
$$

The interpolation function contains the product of the radial $P^{s}(s)$ and transverse $P^{t}(t)$ polynomial functions and a geometric factor to satisfy the Sommerfeld radiation condition. According to Shirron and Babuška, ${ }^{5}$ the interpolation function in the two-dimensional case is defined as

$$
I_{l}(s, t)=\sqrt{\frac{(1-s)}{2}} P_{l_{t}}^{t}(t) P_{l_{s}}^{s}(s),
$$

such that $l=1 \ldots n_{\mathrm{tr}} \cdot n_{\mathrm{rad}}$, where $l_{t}=1 \ldots n_{\mathrm{tr}}$ is an index for the transverse interpolation polynomials and $l_{s}=1 \ldots n_{\text {rad }}$ is an index for the radial interpolation polynomials.

Investigations by von Estorff and Dreyer et al. ${ }^{6,34}$ prove poor suitability of Lagrangian polynomials for higher orders and achieve better performance with Legendre and Jacobi polynomials. This was shown by calculating the condition number of the dynamic stiffness 
matrix $\mathbf{A}$ in the system of linear equations in Eq. (2)

$$
\mathbf{A}(\omega) \mathbf{p}(\omega)=\left(\mathbf{K}-i k \mathbf{D}-k^{2} \mathbf{M}\right) \mathbf{p}(\omega)=\mathbf{f}(\omega)
$$

Solving for the column vector $\mathbf{p}$ gives the unknown nodal pressure values $p_{k}$, which can be used to construct a continuous trial solution $\bar{p}(\mathbf{x}, \omega)$

$$
\bar{p}(\mathbf{x}, \omega) \approx \sum_{k=1}^{N} p_{k}(\omega) \phi_{k}(s, t, \omega)=\mathbf{p}(\omega) \Phi(s, t, \omega)
$$

with the infinite element basis functions $\phi_{k}(s, t, \omega)$ that are given in Eq. (7).

For conjugated Astley-Leis elements, ${ }^{31}$ the test functions $\bar{q}(s, t, \omega)$ use the complex conjugates of the basis functions with an additional geometric weighting factor $(a / r)^{2}=$ $(1-s)^{2} / 4$ such that

$$
\bar{q}(\mathbf{x}, \omega) \approx\left(\frac{1-s}{2}\right)^{2} \mathbf{p}(\omega) \Phi^{*}(s, t, \omega) .
$$

Taking the complex conjugates of the basis functions in the trial and test functions leads to the canceling of the frequency-dependent exponential term in Eq. (7) when the variational statement is formulated. The obtained stiffness, damping and mass matrices of the system are thus independent of frequency.

\subsection{Choice of radial polynomials}

Whereas in early infinite element concepts $^{35,36}$ solely Lagrangian polynomials were used for the radial interpolation functions, Legendre and Jacobi polynomials were found to be a good alternative in terms of improved matrix conditioning and performance. ${ }^{3,5,6}$

\subsubsection{Lagrange polynomials}

Lagrange polynomials are often applied as shape functions in FEM and were used in early concepts of Astley-Leis infinite elements, but led to ill-conditioned system matrices due to oscillatory behavior at high radial orders. ${ }^{6}$ They are given by

$$
P_{j}^{s}(s)=\prod_{\substack{k=1 \ldots N \\ k \neq j}} \frac{s-s_{k}}{s_{j}-s_{k}}
$$

and satisfy the condition that $P_{j}^{s}\left(s_{i}\right)=\delta_{i j}$, where $\delta_{i j}$ is the Kronecker delta. The transition at the boundary between FE and IFE domain is thus compatible.

\subsubsection{Legendre polynomials}

In order to improve the stability of the infinite element concept, Legendre polynomials $P_{i}^{s}(s)$ are applied such that they are orthogonal with respect to the product

$$
\int_{-1}^{1} P_{i}^{s}(s) P_{j}^{s}(s) \mathrm{d} s=\mu_{i} \delta_{i j}
$$


where $\mu_{i}$ is a given constant. To ensure compatibility with the adjacent Lagrangiandiscretized FE domain, the application of a shift pattern as described by Shirron and Babuška ${ }^{5}$ is required.

\subsubsection{Jacobi polynomials}

As a generalization of the Legendre polynomials, Jacobi polynomials $P_{i}^{s}(s)$ were introduced by Dreyer and von Estorff ${ }^{6}$ as radial interpolation functions for infinite elements. They are orthogonal with respect to the product

$$
\int_{-1}^{1}(1-s)^{\alpha}(1+s)^{\beta} P_{i}^{s}(s) P_{j}^{s}(s) \mathrm{d} s=\mu_{i} \delta_{i j}
$$

and depend on the choice of the parameters $\alpha$ and $\beta$. The product of trial and test functions in the variational statement in the two-dimensional case is of the same form as the orthogonality relationship of the Jacobi polynomials when $\alpha=1$ and $\beta=0$

$$
\Phi_{i}(s, t) \Phi_{j}(s, t)^{*}=I_{i}(s, t) e^{-i k \mu(s, t)} I_{j}(s, t) e^{+i k \mu(s, t)} \sim \frac{(1-s)}{2} P_{i}^{s}(s) P_{j}^{s}(s),
$$

which matches well with the orthogonality property. ${ }^{34}$ The same shift pattern as for Legendre polynomials is applied.

\subsubsection{Shift pattern for polynomials}

The radial interpolation functions have to adapt to the Lagrangian finite-element interpolation. Therefore, the first radial polynomial is chosen to satisfy $P_{1}^{s}(-1)=1$ on the boundary between the FE and IFE domains where $s=-1$. The remaining polynomials have to vanish at the infinite element base $P_{i}^{s}(-1)=0$ with $1<i \leq N$ by application of a constant shift as suggested by Shirron and Babuška ${ }^{5}$

$$
P_{i}^{s}(s)=\left\{\begin{array}{ll}
0, & i=1, \\
\bar{P}_{i}^{s}(s)+1, & i=2 n, \\
\bar{P}_{i}^{s}(s)-1, & i=2 n+1,
\end{array} \quad n \in \mathbb{N},\right.
$$

where $\bar{P}_{i}^{s}(s)$ denotes the unshifted polynomial for radial interpolation. The constant shift is required if Legendre or Jacobi polynomials are chosen.

\section{Normal and Acoustic Radiation Modes in Exterior Acoustics}

\subsection{Normal modes}

Starting from the discrete, linear system of equations in Eq. (2), NMs can be found as the eigenvectors of the state-space formulation

$$
(\mathbf{A}+i k \mathbf{B}) \mathbf{z}=\mathbf{r}
$$


with $\mathbf{r}^{T}=[\mathbf{0},-\mathbf{f}]=\left[\mathbf{0},-i \omega \rho_{f} \boldsymbol{\Theta} \mathbf{v}_{s}\right]$, where the hypermatrices $\mathbf{A}$ and $\mathbf{B}$ are constructed from the stiffness, damping and mass matrices

$$
\mathbf{A}=\left[\begin{array}{cc}
\mathbf{M} & \mathbf{0} \\
\mathbf{0} & -\mathbf{K}
\end{array}\right], \quad \mathbf{B}=\left[\begin{array}{cc}
\mathbf{0} & \mathbf{M} \\
\mathbf{M} & \mathbf{D}
\end{array}\right] \quad \text { and } \quad \mathbf{z}=\left[\begin{array}{c}
-i k \mathbf{p} \\
\mathbf{p}
\end{array}\right]
$$

From the asymmetry of the stiffness and damping matrices, left and right eigenvectors are obtained $^{11}$

$$
(\mathbf{A}-\kappa \mathbf{B}) \mathbf{x}_{z}=\mathbf{0} \quad \text { and } \quad \mathbf{y}_{z}^{T}(\mathbf{A}-\kappa \mathbf{B})=\mathbf{0},
$$

which can be written in terms of the modal matrices $\mathbf{Y}$ and $\mathbf{X}$, which diagonalize $\mathbf{A}$ and $\mathbf{B}$

$$
\begin{aligned}
\mathbf{Y}_{z}^{T} \mathbf{A X}_{z} & =\operatorname{diag}\left(\alpha_{1}, \ldots, \alpha_{2 N-\delta}\right), \\
\mathbf{Y}_{z}^{T} \mathbf{B X}_{z} & =\operatorname{diag}\left(\beta_{1}, \ldots, \beta_{2 N-\delta}\right) .
\end{aligned}
$$

On the resulting diagonals, $\alpha_{j}$ and $\beta_{j}$ can be found, the ratio of which is defined to be the $j$ th NM eigenvalue $\kappa_{j}=\alpha_{j} / \beta_{j}$. Applying an additional scaling of $c_{f} / 2 \pi$ leads to a form in which the imaginary part of $\kappa_{j}$ corresponds to the resonance frequency of the $j$ th mode. ${ }^{10}$ The real part of the NM eigenvalues represents a damping term.

\subsection{Acoustic radiation modes}

The eigenvalue problem of the real part of the frequency-dependent and symmetric impedance matrix $\mathbf{Z}$ leads to the eigenvalues $\lambda_{j}$ and their corresponding right eigenvectors as mode shapes at the surface of solid structures, known as ARMs. ${ }^{9}$ The acoustic impedance matrix can be derived from a quadratic form for the sound power in terms of the nodal velocity vector ${ }^{22}$

$$
P=0.5 \mathbf{v}_{\Gamma}^{T} \Re\{\mathbf{Z}\} \mathbf{v}_{\Gamma}^{*} .
$$

This is derived from the discrete formulation by means of the sound pressure and the structural velocity ${ }^{11}$

$$
P=0.5 \Re\left\{\mathbf{p}_{\Gamma}^{T} \boldsymbol{\Theta}_{\Gamma \Gamma} \mathbf{v}_{\Gamma}^{*}\right\},
$$

where the subscript $\Gamma$ ranges over the degrees of freedom (DOF) associated with the surface of the structure. The calculated sound power thus depends only on the choice of the impedance matrix $\mathbf{Z}$, which is often calculated from the boundary element method (BEM) matrices $\mathbf{G}$ and $\mathbf{H} .^{22}$ The relationship $\mathbf{H p}_{\Gamma}=\mathbf{G v}_{\Gamma}$ is rearranged for $\mathbf{p}_{\Gamma}^{T}$ and then substituted into the discrete expression for the sound power in Eq. (24), so that the real part of the acoustic impedance matrix can be found in the inner of the product of the nodal velocity vectors as given in Eq. (23). Peters et al. ${ }^{16}$ show that $\mathbf{Z}_{R}=\Re\{\mathbf{Z}\}$ is required to be symmetric or - if not the case - can easily be symmetrized.

In this paper, the acoustic impedance matrix is obtained by fully inverting the system matrix A constructed by FEM and IFEM. The discrete system of linear equations (2) is subdivided into the DOF on the boundary of the solid structure (marked with the 
subscript $\Gamma$ ) and those associated with the fluid (marked with the subscript o)

$$
\left[\begin{array}{ll}
\mathbf{A}_{\Gamma \Gamma} & \mathbf{A}_{\Gamma \circ} \\
\mathbf{A}_{\circ \Gamma} & \mathbf{A}_{\circ \circ}
\end{array}\right]\left[\begin{array}{l}
\mathbf{p}_{\Gamma} \\
\mathbf{p}_{\circ}
\end{array}\right]=-i \omega \rho_{f}\left[\begin{array}{ll}
\boldsymbol{\Theta}_{\Gamma \Gamma} & \boldsymbol{\Theta}_{\Gamma \circ} \\
\boldsymbol{\Theta}_{\circ \Gamma} & \boldsymbol{\Theta}_{\circ \circ}
\end{array}\right]\left[\begin{array}{c}
\mathbf{v}_{\Gamma} \\
\mathbf{v}_{\circ} .
\end{array}\right]=\left[\begin{array}{l}
\mathbf{f}_{\Gamma} \\
\mathbf{f}_{\circ} .
\end{array}\right] .
$$

Written out in full and considering that there is no structural velocity in the fluid (i.e. $\mathbf{v}_{\circ}=0$ ) gives

$$
\begin{gathered}
\mathbf{A}_{\Gamma \Gamma} \mathbf{p}_{\Gamma}+\mathbf{A}_{\Gamma \circ} \mathbf{p}_{\circ}=-i \omega \rho_{f} \Theta_{\Gamma \Gamma} \mathbf{v}_{\Gamma}, \\
\mathbf{A}_{\circ \Gamma} \mathbf{p}_{\Gamma}+\mathbf{A}_{\circ \circ} \mathbf{p}_{\circ}=-i \omega \rho_{f} \boldsymbol{\Theta}_{\circ \Gamma} \mathbf{v}_{\Gamma} .
\end{gathered}
$$

Solving the latter equation (27) for the sound pressure in the fluid $\mathbf{p}_{\circ}$ leads to

$$
\mathbf{p}_{\circ}=\mathbf{A}_{\circ \circ}^{-1}\left[-i \omega \rho_{f} \boldsymbol{\Theta}_{\circ \Gamma} \mathbf{v}_{\Gamma}-\mathbf{A}_{\circ \Gamma} \mathbf{p}_{\Gamma}\right],
$$

which is then substituted in Eq. (26) and rearranged, so that

$$
\left[\mathbf{A}_{\Gamma \Gamma}-\mathbf{A}_{\Gamma \circ} \mathbf{A}_{\circ \circ}^{-1} \mathbf{A}_{\circ \Gamma}\right] \mathbf{p}_{\Gamma}=-i \omega \rho_{f}\left[\boldsymbol{\Theta}_{\Gamma \Gamma}-\mathbf{A}_{\Gamma \circ} \mathbf{A}_{\circ \circ}^{-1} \boldsymbol{\Theta}_{\circ \Gamma}\right] \mathbf{v}_{\Gamma}
$$

This expression has to be rearranged in order to substitute $\mathbf{p}_{\Gamma}^{T}$ in the equation for the discrete sound power in Eq. (24). It follows that the vector transpose of the nodal sound pressure values on the surface is given by

$$
\mathbf{p}_{\Gamma}^{T}=-i \omega \rho_{f} \mathbf{v}_{\Gamma}^{T}\left[\boldsymbol{\Theta}_{\Gamma \Gamma}-\mathbf{A}_{\Gamma \circ} \mathbf{A}_{\circ \circ}^{-1} \Theta_{\circ \Gamma}\right]^{T}\left[\mathbf{A}_{\Gamma \Gamma}-\mathbf{A}_{\Gamma \circ} \mathbf{A}_{\circ \circ}^{-1} \mathbf{A}_{\circ \Gamma}\right]^{-T} .
$$

Chen and Ginsberg ${ }^{37}$ have shown that the vectors of nodal velocities are extracted by the real part operator in the quadratic form of the sound power in Eq. (23), so that $\mathbf{Z}_{R}$ is given by

$$
\mathbf{Z}_{R}=\Re\{\mathbf{Z}\}=\Re\left\{-i \omega \rho_{f}\left[\boldsymbol{\Theta}_{\Gamma \Gamma}-\mathbf{A}_{\Gamma \circ} \mathbf{A}_{\circ \circ}^{-1} \boldsymbol{\Theta}_{\circ \Gamma}\right]^{T}\left[\mathbf{A}_{\Gamma \Gamma}-\mathbf{A}_{\Gamma \circ} \mathbf{A}_{\circ \circ}^{-1} \mathbf{A}_{\circ \Gamma}\right]^{-T} \boldsymbol{\Theta}_{\Gamma \Gamma}\right\} .
$$

As mentioned above, this impedance matrix is often calculated with the BEM with $\mathbf{Z}_{R}=$ $\Re\left\{\mathbf{H}^{-1} \mathbf{G} \boldsymbol{\Theta}\right\}$, e.g. as done by Peters et al. ${ }^{16}$

\section{Model and Numerical Implementation}

\subsection{Circle}

The purpose of calculating this first model is to prove the reliability of the chosen approach using ARMs. The air surrounding a circle of diameter $0.2 \mathrm{~m}$ is built and meshed with COMSOL Multiphysics using triangular Langrangian finite elements of quadratic order. The air has a density of $\rho_{f}=1.3 \mathrm{~kg} \mathrm{~m}^{-1}$ and a speed of sound of $c_{f}=340 \mathrm{~ms}^{-1}$. After application of FEM and IFEM, the system matrix $\mathbf{A}$ is obtained, which is subdivided (Eq. (25)) and rearranged in order to calculate the acoustic impedance matrix from Eq. (31). The results of the eigenvalue problem of $\mathbf{Z}_{R}$ are presented in Sec. 5.1.

\subsection{Recorder}

In this paper, the authors adapt the model of a recorder by Retka et $a .^{20}$ to the twodimensional case. Therefore, a circular fluid-filled domain $\left(\rho_{f}=1.3 \mathrm{~kg} \mathrm{~m}^{-1}, c_{f}=340 \mathrm{~ms}^{-1}\right)$ 


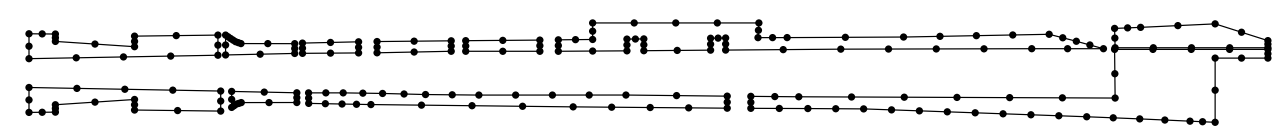

Fig. 1. Boundary mesh nodes at the recorder surface.

with a diameter of $0.67 \mathrm{~m}$ containing the cross-section of the recorder is meshed with COMSOL Multiphysics. Three different mesh sizes are investigated, such that 211 nodes on the recorder surface are shared by all three configurations. These mutual boundary nodes are depicted in Fig. 1. The meshes will be called coarse, normal and fine. The normal mesh consists of 1021 finite elements with a maximum element size of $h_{\max }=3.78 \mathrm{~cm}$ to ensure reliable results at about $3 \mathrm{kHz}$ to $4 \mathrm{kHz} \cdot{ }^{38,39}$ The coarse and the fine mesh consist of 707 and 2102 elements, respectively, with maximum element sizes of $h_{\max }=11.33 \mathrm{~cm}$ and $h_{\max }=2.27 \mathrm{~cm}$. The total numbers of DOF for the sound pressure are 1569, 2197 and 4368 for the coarse, normal and fine meshes, respectively. The FE system matrices of stiffness, damping and mass are imported to Matlab, where the IFEM and subsequent studies are implemented. Mapped and conjugated Astley-Leis infinite elements are attached to the 56 (coarse), 56 (normal) and 60 (fine) elements of the outer boundaries of the three different FE meshes. The Lagrangian interpolation polynomials in the transverse direction inherit quadratic order from the finite elements along the circular FE-IFE-junction. In this paper, the influence of the IFEM on normal and ARMs is investigated. For this purpose, the IFEM system matrices are calculated for different radial interpolation polynomials of varying polynomial degree. Lagrange, Legendre and Jacobi ${ }^{(1,0)}$ as well as Jacobi ${ }^{(2,0)}$ polynomials with orders of 2 up to 20 are used with the normal FE mesh. In the case of the coarse and fine meshes, the subsequent investigations are confined to Jacobi ${ }^{(1,0)}$ polynomials, since the same results are observed for all the radial interpolation polynomials as will be shown in the following results section. The radial interpolation points are positioned equidistantly between $[-1,1)$ in the direction of the mapped radial coordinate $s$, such that the first interpolation point is located at $a=0.33 \mathrm{~m}$ on the circular junction of the FE and the IFE domain. According to the infinite element mapping approach in Eq. (6), the outer interpolation points in the global coordinate system can be found at the radii $r_{j}$ in [m] for $j=2 \ldots 20$ : $[0.67,1,1.33,1.67,2,2.33,2.67,3,3.33,3.67,4,4.33,4.67,5,5.33,5.67,6,6.33,6.67]$.

The subsequent considerations are made in the frequency range $1 \mathrm{~Hz}-3000 \mathrm{~Hz}$ in frequency steps of $1 \mathrm{~Hz}$.

\section{Results}

\subsection{Circle}

For the circle in air, the radiation modes of the acoustic impedance matrix are computed according to the derivation made in Sec. 3.2. In Fig. 2, the nine largest ARM eigenvalues $\lambda_{j}$ are depicted as functions of the frequency. The largest eigenvalue $\left(\lambda_{1}:-\right)$ corresponds to a monopole mode and subsequent modes appear as pairs of eigenvalues of equal magnitude, corresponding to dipoles $\left(\lambda_{2}\right.$ and $\left.\lambda_{3}:----\right)$, quadrupoles $\left(\lambda_{4}\right.$ and $\left.\lambda_{5}:-\cdot-\cdot-\right)$, hexapoles $\left(\lambda_{6}\right.$ 


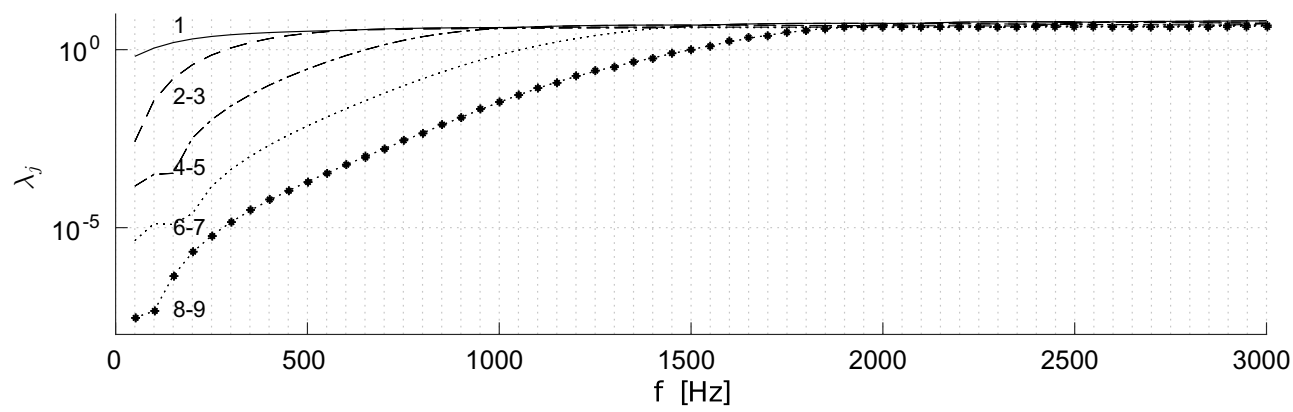

Fig. 2. The nine largest radiation mode eigenvalues $\lambda_{j}$ as functions of the frequency, shown in groups of multipoles.
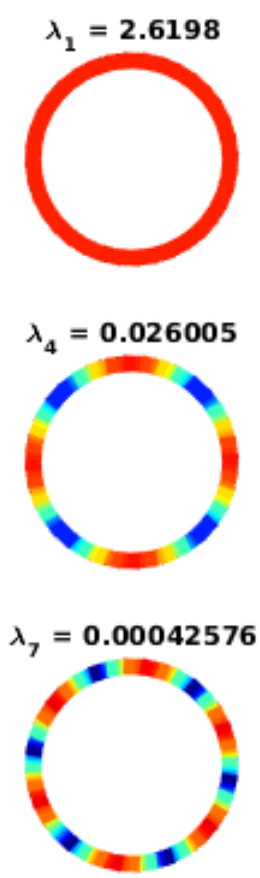
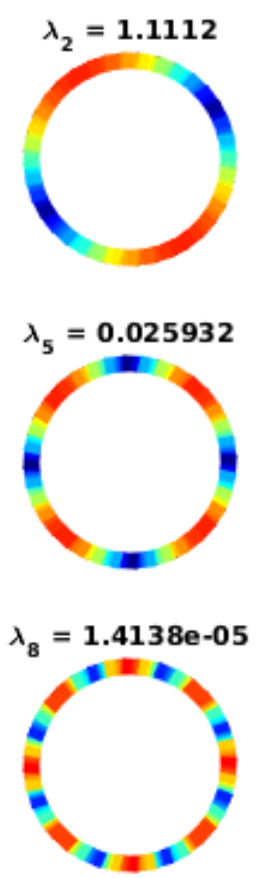

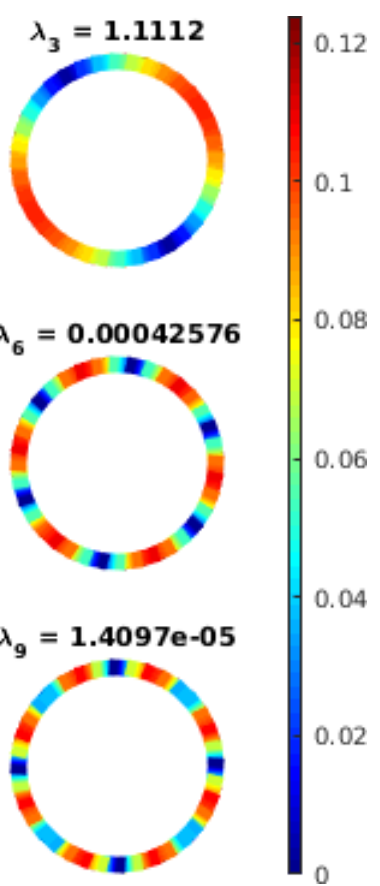

0.1

.08

06

04

02

Fig. 3. ARM mode shapes corresponding to the nine largest eigenvalues $\lambda_{j}$ at $300 \mathrm{~Hz}$.

and $\left.\lambda_{7}: \cdots\right)$ ) as well as octupoles $\left(\lambda_{8}\right.$ and $\left.\lambda_{9}: \cdot * * \cdot\right)$, depicted in Fig. 3. The grouping behavior of ARM eigenvalues or radiation efficiencies in terms of acoustical multipoles has already been presented by Cunefare et $a .^{15}$ for three-dimensional problems and by $\mathrm{Wu}$ et al. ${ }^{40}$

\subsection{Recorder}

\subsubsection{Matrix condition number}

As a first step, the condition number of the dynamic stiffness matrix $\mathbf{A}$ (cf. Eq. (10)) is calculated. The condition number $\kappa(\mathbf{A})$ is given by the ratio of the largest to the lowest 
eigenvalue of the frequency-dependent matrix $\mathbf{A}$ and is calculated by power iteration and inverse iteration, respectively. As shown by Babuška et al. ${ }^{41}$ Cremers et al. ${ }^{42}$ and Dreyer et $a l .{ }^{6}$ the matrix condition increases with the polynomial order, which leads to ill-conditioned matrices for polynomials of degree $>8$ in the case of Lagrange polynomials, whereas Legendre and Jacobi polynomials provide distinctly better-conditioned matrices. Tests performed by the authors of this paper have confirmed this observation, although the choice between the four polynomials had no considerable effect on the modes in exterior acoustics, as will be shown subsequently.

\subsubsection{Influence of infinite elements on radiation modes}

The eigenvalues $\lambda_{j}$ and eigenvectors of the frequency-dependent, real acoustic impedance matrix $\mathbf{Z}_{R}$ from Eq. (31) were computed separately for each frequency step. The largest eigenvalues as a function of the frequency are shown in Fig. 4 for the four polynomials of degree 20 with the normal FE mesh. All polynomials exhibit virtually identical eigenvalue curves and do so for each degree of radial interpolation, not only for the 20th order. Despite the differences in matrix condition, the choice of polynomial for radial interpolation does not seem to matter for calculating the ARM.

In Fig. 4, the grouping behavior of the exterior radiation mode eigenvalues can be observed as described by Cunefare et al. ${ }^{15}$ and by Wu et $a l .{ }^{40}$ and as obtained for the circle in Sec. 5.1, which is a pure exterior acoustic problem without holes or chambers with interior modes. These grouped curves are in superposition with interior resonances in the hollow recorder. This is indicated by single eigenvalues that show resonance peaks at certain frequencies above which they rapidly decrease, which leads to changes in the order of eigenvalue magnitudes. The distribution of eigenvalues at each frequency is related to the share of the corresponding mode shape in the total superimposed pressure field at the surface of the solid structure, since ARM eigenvalues are related to the radiation

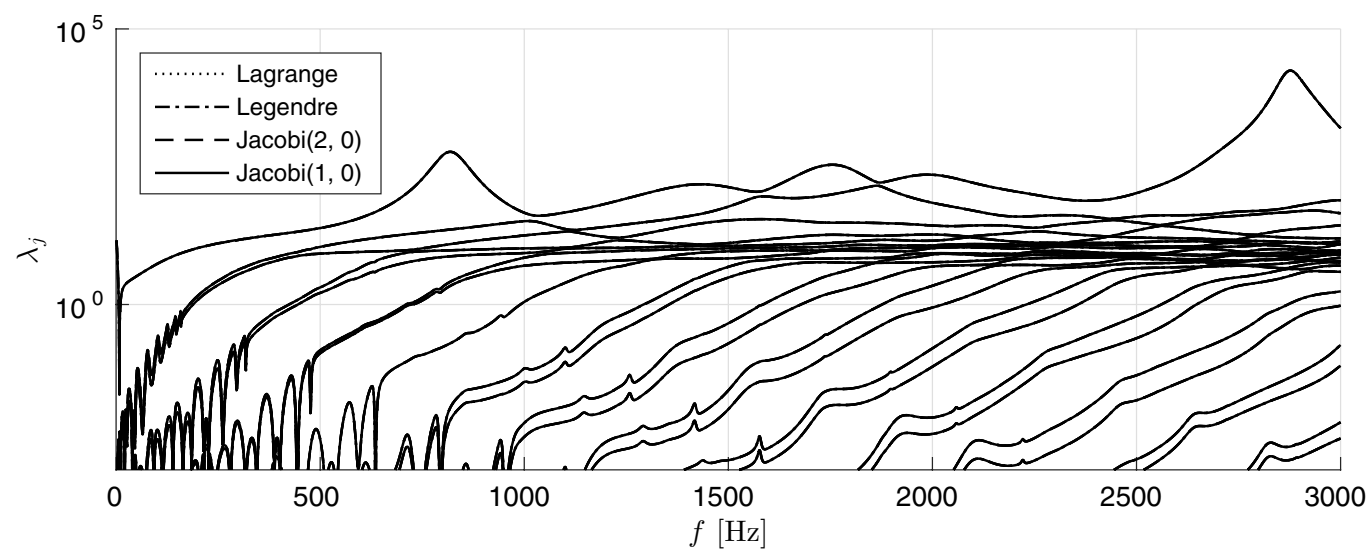

Fig. 4. Largest eigenvalues $\lambda_{j}$ of the radiation modes as a function of the frequency for Lagrange, Legendre and two Jacobi polynomials of radial order 20 with the normal FE mesh. All polynomials lead to virtually identical eigenvalue curves. 


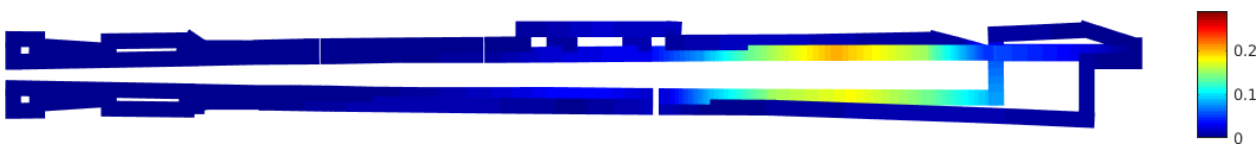

(a) $\lambda_{1}$ at $840 \mathrm{~Hz}$

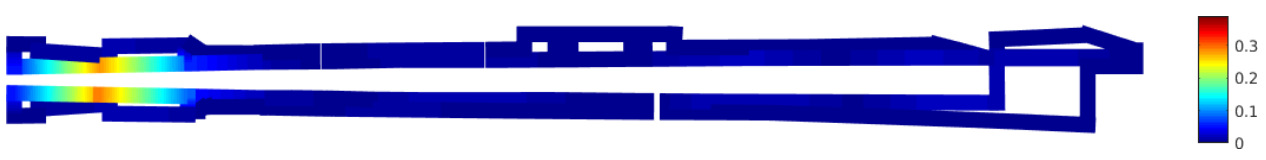

(b) $\lambda_{1}$ at $1760 \mathrm{~Hz}$

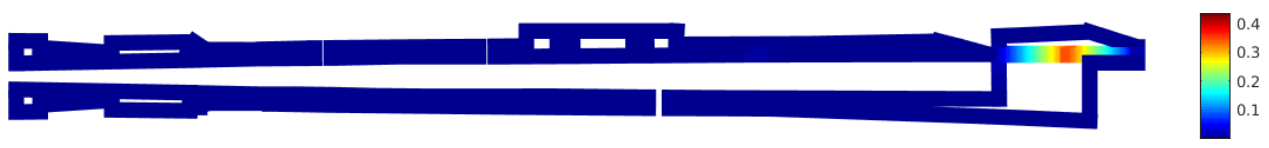

(c) $\lambda_{1}$ at $2880 \mathrm{~Hz}$

Fig. 5. ARM shapes as eigenvectors of the acoustic impedance matrix corresponding to the largest eigenvalue at each of the given frequencies.

efficiency $\sigma$ by $\lambda=\rho_{f} c_{f} \sigma .{ }^{16}$ Three notable peaks can be found for the largest eigenvalue at approximately $840 \mathrm{~Hz}, 1760 \mathrm{~Hz}$ and $2880 \mathrm{~Hz}$. The corresponding mode shapes — which can be found as eigenvectors of the acoustic impedance matrix $\mathbf{Z}_{R}$ - are depicted in Fig. 5 and show interior modes in the upper middle section (barrel), see Fig. 5(a), in the foot joint (Fig. 5(b)) and in the windway of the mouthpiece (Fig. 5(c)).

In the following, attention will be restricted to one single type of IFEM interpolation polynomial in the radial direction, the $\operatorname{Jacobi}(1,0)$ polynomials. As shown before, the same results for the ARMs are obtained for all considered polynomials. Using the Jacobi(1, 0) polynomials leads to the best-conditioned problem due to the lowest matrix condition number $\kappa(\mathbf{A})$. Two further finite element meshes are taken into consideration and the infinite element system matrices are computed for Jacobi $(1,0)$ as radial interpolation polynomials of degree 2 up to 20. The ARM eigenvalues $\lambda_{j}$ with $j=1, \ldots, 8$ with coarse, normal and fine FE mesh are depicted in Fig. 6 for a degree of 20. The results show that the coarse and normal meshes lead to quite similar eigenvalue curves, whereas the fine FE mesh results in slightly smaller values of ARM eigenvalues.

Close to the eigenvalue resonance peaks it can be observed that each single eigenvalue converges as the degree of the radial interpolation polynomials increases, as can be seen in Fig. 7 in the example of the peak in $\lambda_{1}$ at about $2880 \mathrm{~Hz}$. Polynomials of even and odd degrees respectively lead to curves that converge to two different eigenvalues. They do not necessarily approach from opposite sides, but sometimes both from below or from above. However, away from the resonance peaks, the difference between eigenvalue curves of low-order polynomials and the curves of the highest-order converged polynomials with degree 19 and 20 is negligible. This means that the loss of accuracy incurred by reducing the degree of radial interpolation polynomials only emerges at the modal resonances. In 


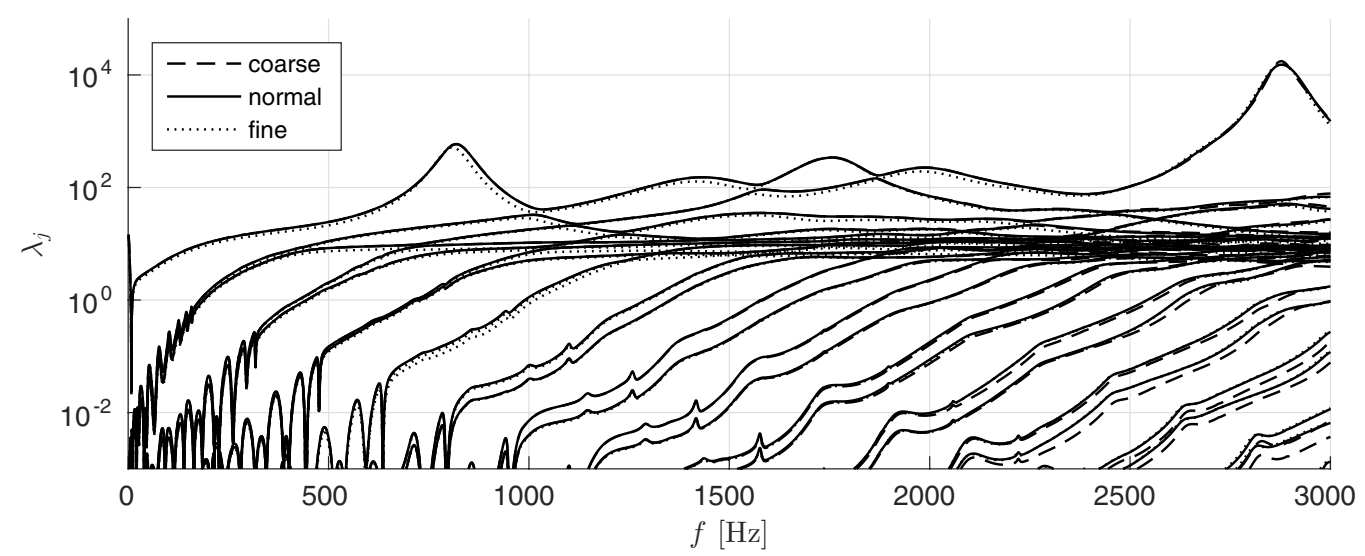

Fig. 6. Radiation mode eigenvalues $\lambda_{j}$ for $\operatorname{Jacobi}(1,0)$ interpolation polynomials of degree 20 for coarse, normal and fine FE mesh.

addition, convergence of the eigenvalues from both sides can be observed, depending on the FE mesh on which the computation is based. In the example of the peak considered earlier at about $2880 \mathrm{~Hz}$, the eigenvalues obtained from IFEM radial interpolation polynomials with even degree converge from below for the coarse FE mesh (see Fig. 7(a)), whereas calculations based on the normal and the fine mesh lead to convergence from above, as can be seen in Figs. 7(b) and 7(c). The convergence of eigenvalues from odd-degree polynomials is observed to behave vice versa at this particular resonance. However, at least once, the curves cross and converge from opposite sides close to the resonance peaks.

\subsubsection{Influence of infinite elements on $N M$ s}

Transforming the frequency-independent system matrices $\mathbf{K}, \mathbf{D}$ and $\mathbf{M}$ into the state-space form as given in Eq. (19) leads to the eigenvalue problem in Eq. (18) that yields the NMs as right eigenvectors. The corresponding eigenvalues $\kappa_{j}$ are complex numbers with frequency information in the imaginary part and a damping term in the real part. Figure 8 shows the eigenvalues in the complex plane for all four polynomials at radial interpolation of degree $n_{\text {rad }}=10$ and $n_{\text {rad }}=20$ with the normal FE mesh. The focus is on eigenvalues with a positive frequency below $3 \mathrm{kHz}$ (neglecting the complex conjugate eigenvalues) whose real part has comparatively small magnitude $\left(\left|\Re\left\{\kappa_{j}\right\}\right|<100\right)$, which means the considered modes are weakly damped and thus expected to have a major influence on the radiation of sound. Clearly, the eigenvalue solution does not depend on the choice of the radial interpolation polynomial for the infinite elements, since Lagrange, Legendre and both Jacobi polynomials lead to virtually the same eigenvalues in the complex plane as shown in Fig. 8. Since this parameter has no influence on the NM eigenvalues, only Jacobi $(1,0)$ polynomials are taken into consideration in the following.

Single eigenvalues or a certain number of eigenvalues on a curve can be observed, which can be recognized in all of the calculations, independent of the number of radial interpolation points $n_{\text {rad }}$. These eigenvalues are expected to converge as the order of the polynomials 


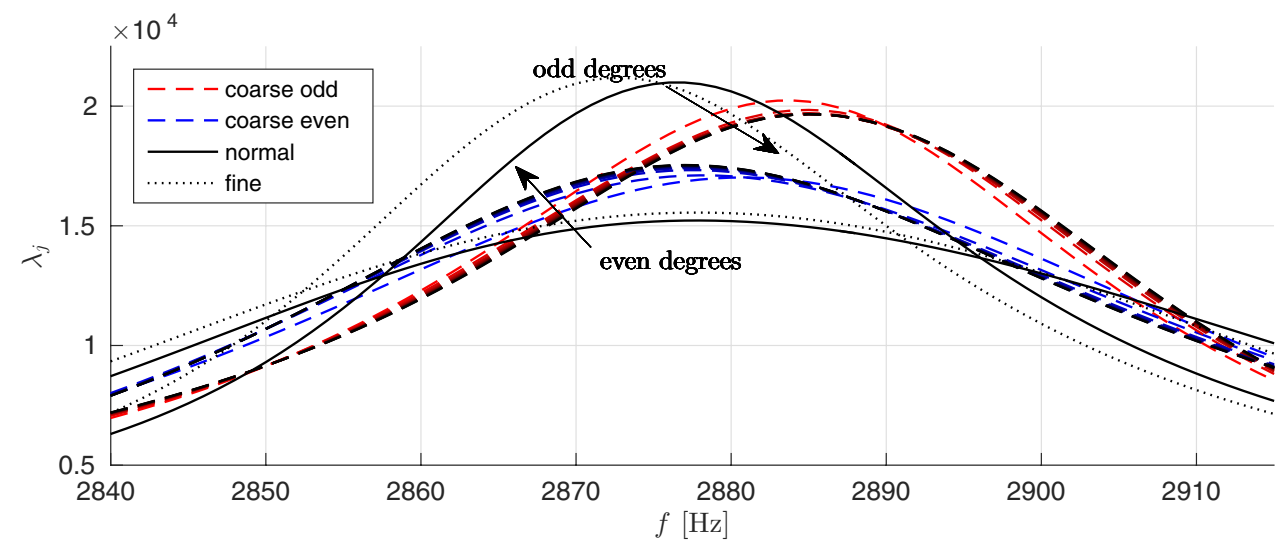

(a) ARM eigenvalues $\lambda_{j}$ based on coarse FE mesh

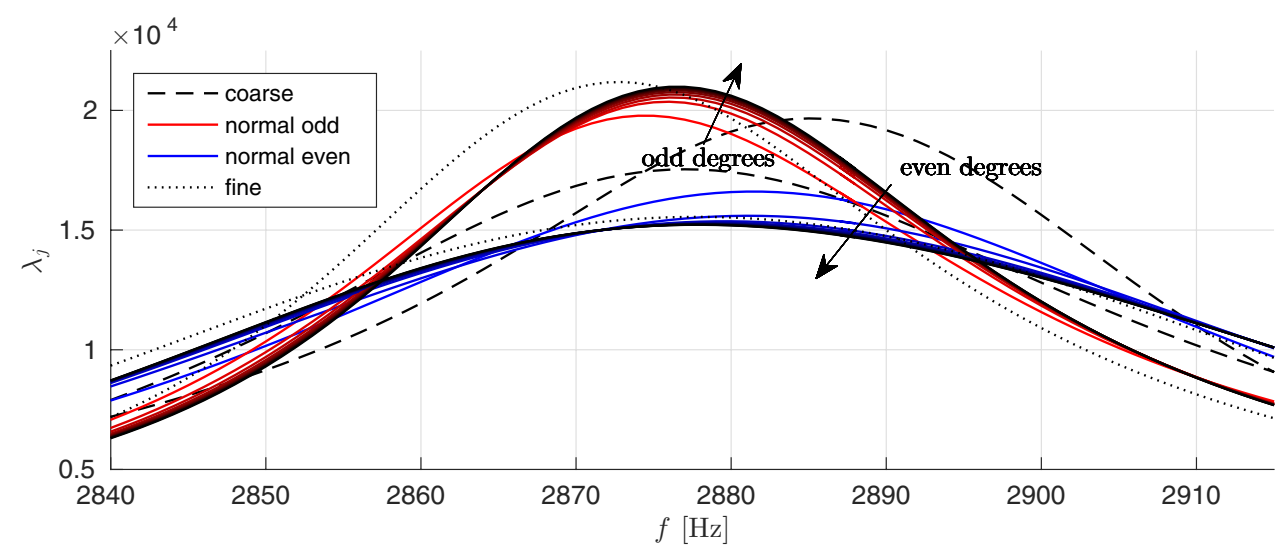

(b) ARM eigenvalues $\lambda_{j}$ based on normal FE mesh

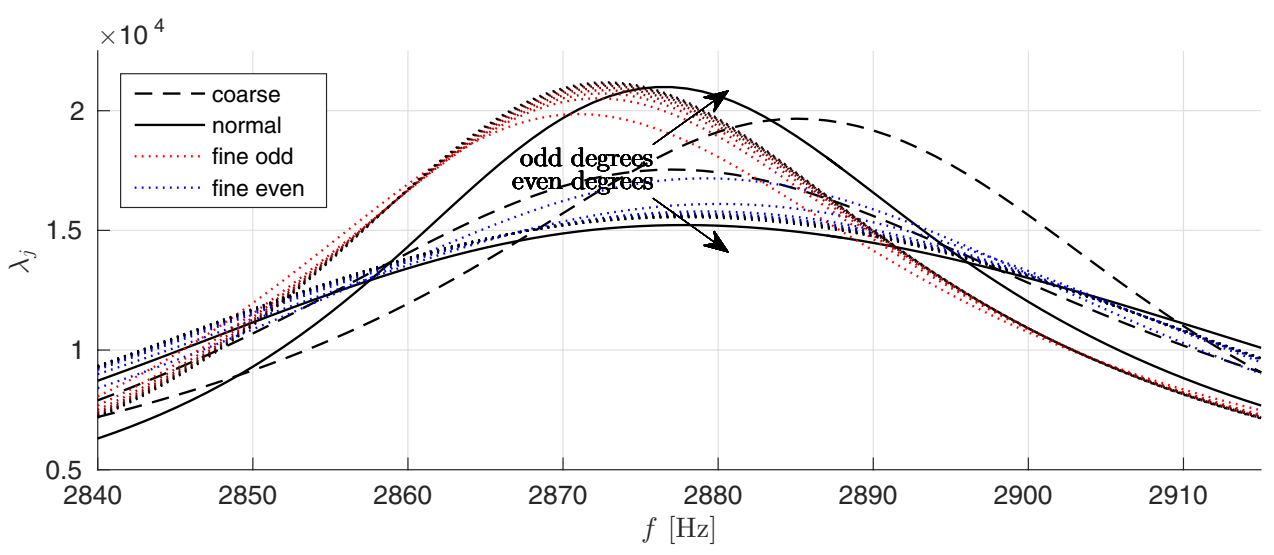

(c) ARM eigenvalues $\lambda_{j}$ based on fine FE mesh

Fig. 7. (Color online) Convergence of ARM eigenvalues $\lambda_{j}$ based on three different FE meshes (coarse - -- , normal — and fine .....). Polynomials of even and odd degrees are denoted with blue and red lines, respectively. The black curves - in pairs, one for even and one odd order — denote the converged eigenvalues of degree 19 or 20 for all FE meshes. 


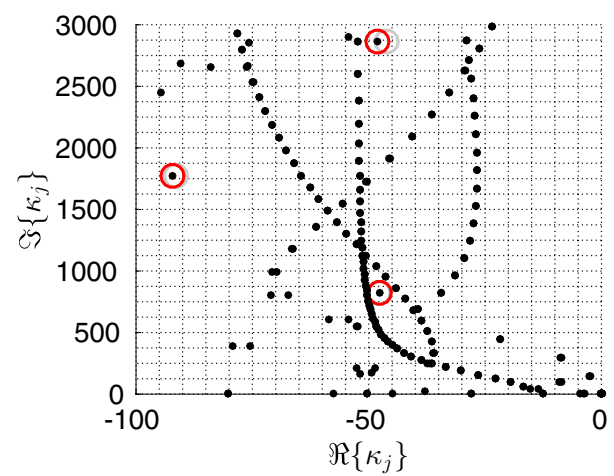

(a) $\kappa_{j}$ at $n_{\text {rad }}=10$

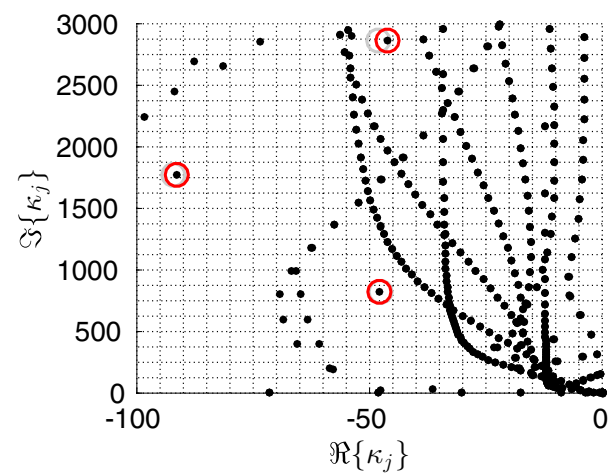

(b) $\kappa_{j}$ at $n_{\text {rad }}=20$

Fig. 8. (Color online) Complex plane with NM eigenvalues for Lagrange, Legendre, Jacobi(2, 0) and Jacobi(1, $0)$ polynomials, which all give virtually the same eigenvalues depicted as black dots. Radial interpolation polynomial of: (a) degree 10 and (b) degree 20. The red circles show the three related eigenvalues that were found for the ARMs in Figs. 4 and 5. The gray circles indicate the variation of the considered eigenvalues (red circles) between the polynomial degrees 10 and 20 .

increases, whereas the additional eigenvalues that appear due to the growing number of DOF do not converge and are assumed to correspond to spurious modes. This was also observed by Marburg ${ }^{11}$ when he investigated NMs in the fluids around an ellipse and an open box, respectively. The red circles indicate three eigenvalues $\kappa_{a}, \kappa_{b}$ and $\kappa_{c}$ which correspond to eigenvectors or mode shapes that were found in resonance at approximately $840 \mathrm{~Hz}$ $\left(\lambda_{1}\right), 1760 \mathrm{~Hz}\left(\lambda_{1}\right)$ and $2880 \mathrm{~Hz}\left(\lambda_{1}\right)$ for the ARMs (cf. Fig. 4). Almost the same three frequencies can be found in the imaginary parts of the three red-marked NM eigenvalues $\kappa_{13}=-46.22+819.27 i\left(=\kappa_{a}\right), \kappa_{72}=-98.01+1743.25 i\left(=\kappa_{b}\right)$ and $\kappa_{186}=-38.78+2880.84 i$ $\left(=\kappa_{c}\right)$ in the example of second-order Jacobi $(1,0)$ polynomials with the normal FE mesh. This single value in the imaginary part of the complex eigenvalues shows the frequency at which the respective NM is in resonance, whereas its damping ratio is given in the real part as described by Marburg. ${ }^{10}$ The corresponding mode shapes in the fluid around the recorder - as right eigenvectors of the state space eigenvalue problem - are depicted in Fig. 9. As expected, the same mode shapes can be obtained in the barrel and in the mouthpiece of the recorder as for the ARMs in Fig. 5.

Similar to the eigenvalues of the ARMs, the NM eigenvalues differ for radial interpolation polynomials of even and odd degrees. This means that two different convergent complex conjugate pairs of eigenvalues are calculated, corresponding to the same mode shape. By application of the Modal Assurance Criterion (MAC), ${ }^{29}$ the NM eigenvalues can be tracked throughout all the calculations. The MAC can be calculated by ${ }^{29}$

$$
\operatorname{MAC}(\mathbf{a}, \mathbf{b})=\frac{\left|\mathbf{a}^{H} \mathbf{b}\right|^{2}}{\left(\mathbf{a}^{H} \mathbf{a}\right)\left(\mathbf{b}^{H} \mathbf{b}\right)},
$$

where $\mathbf{a}$ and $\mathbf{b}$ are eigenvectors or columns of modal matrices and $(\cdot)^{H}=(\cdot)^{\prime *}$ is the Hermitian transpose (complex conjugate and transpose). The comparison of modal matrices 


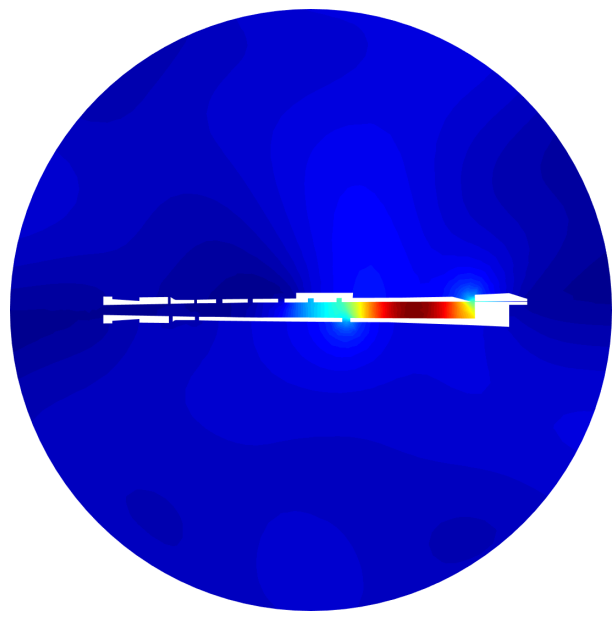

(a) $\kappa_{a}=\kappa_{13}=-46.22+819.27 i$

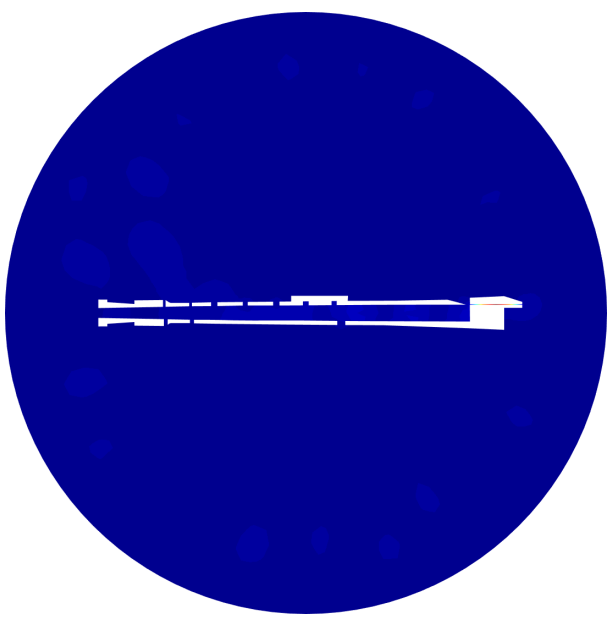

(c) $\kappa_{c}=\kappa_{186}=-38.78+2880.84 i$

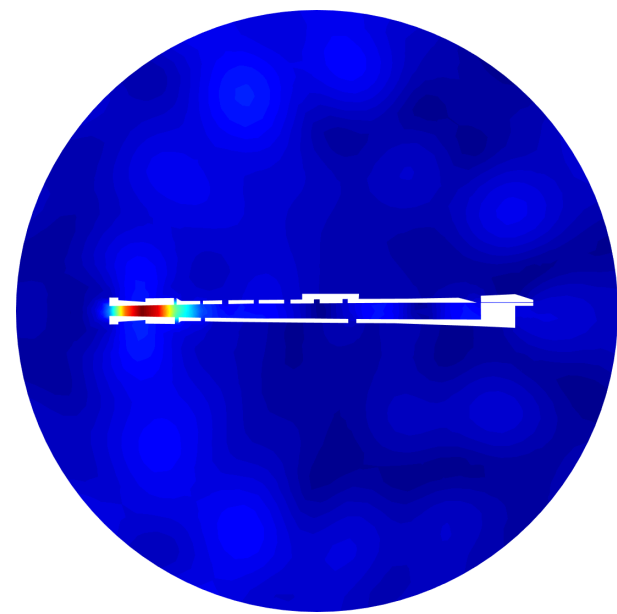

(b) $\kappa_{b}=\kappa_{72}=-98.01+1743.25 i$

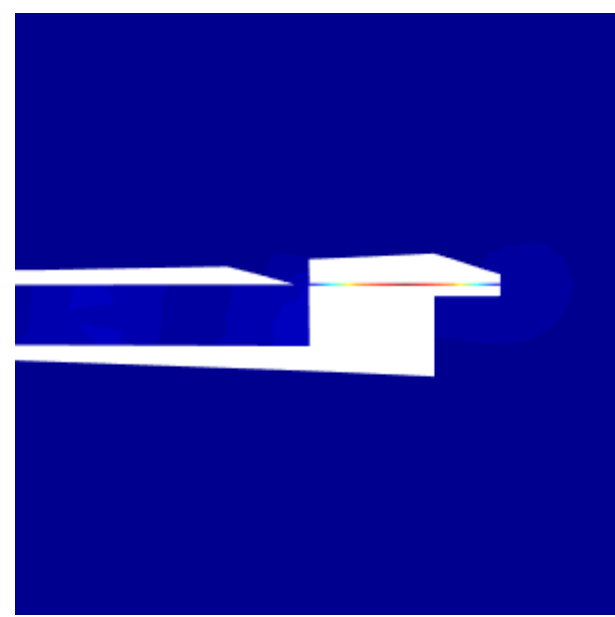

(d) Enlargement of the mouthpiece for $\kappa_{c}$

Fig. 9. NM shapes as right eigenvectors corresponding to given complex eigenvalues ((a) $\kappa_{13}$, (b) $\kappa_{72}$, (c) and $\left.(\mathrm{d}) \kappa_{186}\right)$ for Jacobi $(1,0)$ polynomials of second-order of radial interpolation, based on the normal $\mathrm{FE}$ mesh.

leads to a MAC matrix, whose maximum value(s) denote(s) the best accordance of two compared eigenvectors. The positions of the maxima indicate the corresponding eigenvalues of well-according mode shapes. The eigenvectors that are depicted in Fig. 9 (normal FE mesh and second-order interpolation polynomials) are compared to each of the remaining eigenvectors that were calculated with varying polynomial order based on three FE meshes. To ensure comparability between all modal matrices, eigenvectors are compared, which are narrowed to the mutual DOF on the recorder surface (211 DOF), cf. Sec. 4.2. Since the three tracked modes only have notable peaks in the interior and therefore directly at the surface of the recorder, this is a reasonable reduction. 


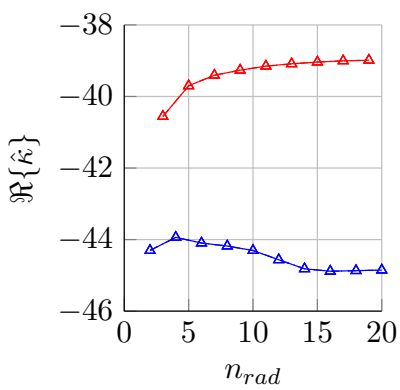

(a) $\Re\left\{\kappa_{a}\right\}$ as function of $n_{\text {rad }}$

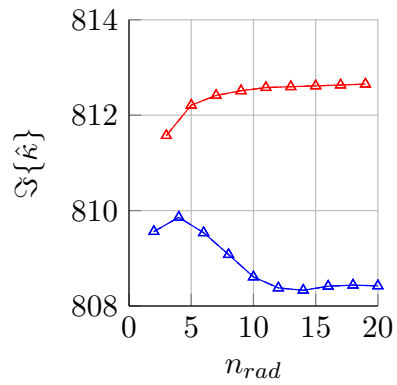

(d) $\Im\left\{\kappa_{a}\right\}$ as function of $n_{\text {rad }}$

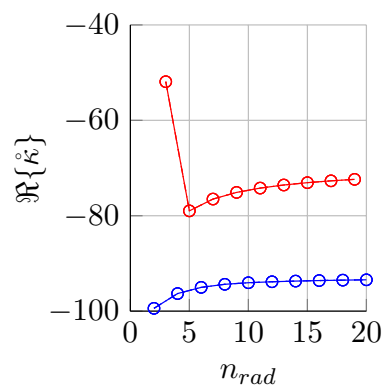

(b) $\Re\left\{\kappa_{b}\right\}$ as function of $n_{\text {rad }}$

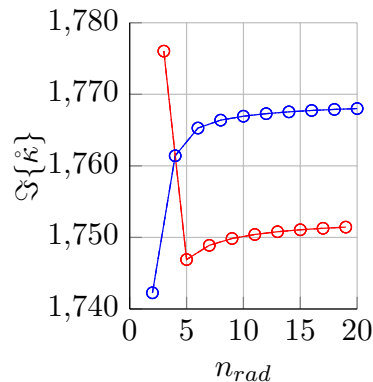

(e) $\Im\left\{\kappa_{b}\right\}$ as function of $n_{\text {rad }}$

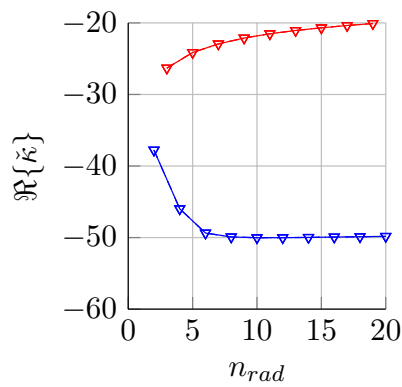

(c) $\Re\left\{\kappa_{c}\right\}$ as function of $n_{\text {rad }}$

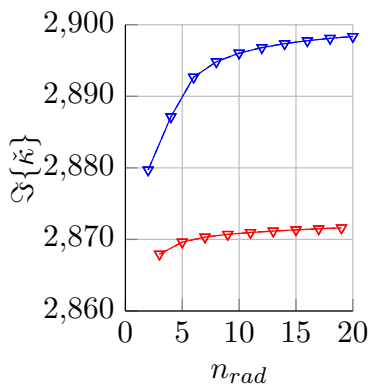

(f) $\Im\left\{\kappa_{c}\right\}$ as function of $n_{\mathrm{rad}}$

Fig. 10. (Color online) Convergence of the eigenvalues $\kappa_{a}(\triangle), \kappa_{b}(\bigcirc)$ and $\kappa_{c}(\nabla)$ as functions of even (blue) and odd (red) polynomial order for radial interpolation $n_{\text {rad }}$ with three different FE meshes (coarse - - - normal $\longrightarrow$ and fine....$)$, whose curves are virtually the same.

Figure 10 shows the convergence of the NM eigenvalues $\kappa_{a}, \kappa_{b}$ and $\kappa_{c}$ as the degree of the radial interpolation polynomials of the infinite elements is increased and considering the three different FE meshes. It can be seen that the choice of coarse, normal or fine FE mesh has hardly any effect, neither on the real part nor on the imaginary part of the converging eigenvalues. As already observed for the ARM eigenvalues, two different convergence curves of the NM eigenvalues for polynomials of even and odd orders are found.

\section{Conclusion}

In this work, the authors applied the FEM as well as the IFEM in order to discretize and calculate the spatial sound pressure field in fluid-filled unbounded domains around an inner solid structure. The obtained discrete system matrices were used for modal decomposition into ARMs and NMs. It was shown that the acoustic impedance matrix $\mathbf{Z}_{R}$ for the calculation of the radiation modes can also be determined from the matrices of the finite and the infinite elements, instead of the boundary element matrices that are usually used. Furthermore, the grouping behavior of exterior multipole eigenvalues was observed in the example of a circle and a recorder in a fluid-filled unbounded domain. In addition, resonances of interior radiation mode eigenvalues were found for the hollow model of the recorder. The 


\section{Moheit \&3 S. Marburg}

comparison between the ARMs and the NMs shows that both methods provide almost all the same eigenvectors (mode shapes) with an acceptable agreement for the corresponding frequencies. Radiation mode shapes only refer to the surface of the structure and NM eigenvectors can be visualized in the whole discretized fluid-filled domain.

The aim of this paper was to investigate the influence of FE mesh sizes as well as different IFE radial interpolation polynomials and polynomial degrees on the modes in exterior acoustics. The investigations show no differences, neither for ARM eigenvalues nor for NM eigenvalues, between the choice of Lagrange, Legendre or Jacobi polynomials. However, the matrix condition of the global system matrix $\mathbf{A}$ is the best with Jacobi(1,0) polynomials, so that further investigations were limited to these polynomials. Variation of the finite element mesh size has no considerable effect on the NM eigenvalues and has a minor effect on some of the radiation mode eigenvalues in the whole frequency range. Increasing the degree of radial interpolation polynomials in the domain of the infinite elements reveals two convergence curves for the ARM eigenvalues for polynomials of even and odd degrees. Differences in the eigenvalue curves of lower- and higher-order interpolation polynomials mainly emerge close to the resonance peaks of the eigenvalues rather than over the whole frequency range. The investigations revealed no clear tendency for convergence of the eigenvalues from above or below. In order to track NM eigenvalues for different FE meshes and polynomial degrees, the corresponding eigenvectors were compared to each other by application of the MAC. In the example of three significant NM shapes, the NM eigenvalues were observed to converge as the order of the polynomials was increased. Similar to the convergence of the radiation modes, the authors found two convergences of the NM eigenvalues for polynomials of even and odd degree.

Future work is planned to further investigate the eigenvalue solver and alternative, iterative methods to reduce the calculation time. The role of the eigenvalue solver in the differences in polynomials of even and odd degree has to be clarified. Finally, a modal reduction is desirable in order to calculate the radiated sound power or sound pressure field with a reduced number of modal contributions.

\section{Acknowledgments}

We wish to gratefully acknowledge the support for this research provided by the Deutsche Forschungsgemeinschaft (DFG) under grant number MA 2395/11-1.

\section{References}

1. A. Bermúdez, L. Hervella-Nieto, A. Prieto and R. Rodríguez, Perfectly matched layers, in Computational Acoustics of Noise Propagation in Fluids - Finite and Boundary Element Methods, eds. S. Marburg and B. Nolte (Springer, Berlin, 2008), pp. 167-196.

2. R. J. Astley, G. J. Macaulay, J.-P. Coyette and L. Cremers, Three-dimensional wave-envelope elements of variable order for acoustic radiation and scattering. Part I. Formulation in the frequency domain, J. Acoust. Soc. Am. 103(1) (1998) 49-63.

3. R. J. Astley, Infinite elements for wave problems: A review of current formulations and an assessment of accuracy, Int. J. Numer. Methods Eng. 49(7) (2000) 951-976. 
4. F. Ihlenburg, Finite Element Analysis of Acoustic Scattering, Vol. 132 (Springer Science \& Business Media, 2006).

5. J. J. Shirron and I. Babuška, A comparison of approximate boundary conditions and infinite element methods for exterior Helmholtz problems, Comput. Methods Appl. Mech. Eng. 164(1) (1998) 121-139.

6. D. Dreyer and O. von Estorff, Improved conditioning of infinite elements for exterior acoustics, Int. J. Numer. Methods Eng. 58(6) (2003) 933-953.

7. G. V. Borgiotti, The power radiated by a vibrating body in an acoustic fluid and its determination from boundary measurements, J. Acoust. Soc. Am. 88(4) (1990) 1884-1893.

8. D. M. Photiadis, The relationship of singular value decomposition to wave-vector filtering in sound radiation problems, J. Acoust. Soc. Am. 88(2) (1990) 1152-1159.

9. A. Sarkissian, Acoustic radiation from finite structures, J. Acoust. Soc. Am. 90(1) (1991) $574-$ 578.

10. S. Marburg, Normal modes in external acoustics. Part I: Investigation of the one-dimensional duct problem, Acta Acust. United with Acust. 91(6) (2005) 1063-1078.

11. S. Marburg, F. Dienerowitz, T. Horst and S. Schneider, Normal modes in external acoustics. Part II: Eigenvalues and eigenvectors in 2D, Acta Acust. United with Acust. 92(1) (2006) 97-111.

12. S. Marburg, Normal modes in external acoustics. Part III: Sound power evaluation based on superposition of frequency-independent modes, Acta Acust. United with Acust. 92(2) (2006) 296-311.

13. K. A. Cunefare, The minimum multimodal radiation efficiency of baffled finite beams, J. Acoust. Soc. Am. 90(5) (1991) 2521-2529.

14. K. A. Cunefare and M. N. Currey, On the exterior acoustic radiation modes of structures, J. Acoust. Soc. Am. 96(4) (1994) 2302-2312.

15. K. A. Cunefare, M. N. Currey, M. E. Johnson and S. J. Elliott, The radiation efficiency grouping of free-space acoustic radiation modes, J. Acoust. Soc. Am. 109(1) (2001) 203-215.

16. H. Peters, N. Kessissoglou and S. Marburg, Enforcing reciprocity in numerical analysis of acoustic radiation modes and sound power evaluation, J. Comput. Acoust. 20(3) (2012).

17. H. Wu, W. Jiang and Y. Liu, Analyzing acoustic radiation modes of baffled plates with a fast multipole boundary element method, J. Vib. Acoust. 135(1) (2013) 011007.

18. S. Marburg, E. Lösche, H. Peters and N. Kessissoglou, Surface contributions to radiated sound power, J. Acoust. Soc. Am. 133(6) (2013) 3700-3705.

19. Z.-B. Liu and C. Maury, An improved method for the calculation of near-field acoustic radiation modes, J. Sound Vib. 363 (2016) 316-328.

20. S. Fuß, S. C. Hawkins and S. Marburg, An eigenvalue search algorithm for the modal analysis of a resonator in free space, J. Comput. Acoust. 19(1) (2011) 95-109.

21. S. Retka and S. Marburg, An infinite element for the solution of Galbrun equation, ZAMM J. Appl. Math. Mech./Zeit. Angew. Math. Mech. 93(2-3) (2013) 154-162.

22. K. A. Cunefare and G. H. Koopmann, Global optimum active noise control: Surface and far-field effects, J. Acoust. Soc. Am. 90(1) (1991) 365-373.

23. S. S. Ramesh, K.-M. Lim and B. C. Khoo, Comparison of constant and discontinuous quadratic boundary elements for exterior axisymmetric acoustic-wave propagation problems, J. Comput. Acoust. 23(4) (2015) 1540003.

24. S. Marburg, The Burton and Miller method: Unlocking another mystery of its coupling parameter, J. Comput. Acoust. 24(1) (2016) 1550016.

25. S. Marburg, Numerical damping in the acoustic boundary element method, Acta Acust. United with Acust. 102(3) (2016) 415-418.

26. C.-J. Zheng, H.-B. Chen, H.-F. Gao and L. Du, Is the Burton-Miller formulation really free of fictitious eigenfrequencies?, Eng. Anal. Bound. Elem. 59 (2015) 43-51. 
27. L. Demkowicz and K. Gerdes, Convergence of the infinite element methods for the Helmholtz equation in separable domains, Numer. Math. 79(1) (1998) 11-42.

28. K. Gerdes, A summary of infinite element formulations for exterior Helmholtz problems, Comput. Methods Appl. Mech. Eng. 164(1) (1998) 95-105.

29. R. J. Allemang, The modal assurance criterion-twenty years of use and abuse, Sound Vib. 37(8) (2003) 14-23.

30. S. Marburg and B. Nolte, A unified approach to finite and boundary element discretization in linear timeharmonic acoustics, in Computational Acoustics of Noise Propagation in Fluids Finite and Boundary Element Methods, eds. S. Marburg and B. Nolte (Springer, Berlin, 2008), pp. $1-34$.

31. R. J. Astley, Infinite elements, in Computational Acoustics of Noise Propagation in Fluids Finite and Boundary Element Methods, eds. S. Marburg and B. Nolte (Springer, Berlin, 2008), pp. 197-230.

32. R. J. Astley and J.-P. Coyette, Conditioning of infinite element schemes for wave problems, Commun. Numer. Methods Eng. 17(1) (2001) 31-41.

33. J. M. M. C. Marques and D. R. J. Owen, Infinite elements in quasi-static materially nonlinear problems, Comput. Struct. 18(4) (1984) 739-751.

34. O. von Estorff, S. Petersen and D. Dreyer, Efficient infinite elements based on Jacobi polynomials, in Computational Acoustics of Noise Propagation in Fluids - Finite and Boundary Element Methods, eds. S. Marburg and B. Nolte (Springer, Berlin, 2008), pp. 231-250.

35. R. J. Astley and W. Eversman, Finite element formulations for acoustical radiation, J. Sound Vib. 88(1) (1983) 47-64.

36. O. C. Zienkiewicz, K. Bando, P. Bettess, C. Emson and T. C. Chiam, Mapped infinite elements for exterior wave problems, Int. J. Numer. Methods Eng. 21(7) (1985) 1229-1251.

37. P.-T. Chen and J. H. Ginsberg, Complex power, reciprocity, and radiation modes for submerged bodies, J. Acoust. Soc. Am. 98(6) (1995) 3343-3351.

38. S. Marburg and S. Schneider, Influence of element types on numeric error for acoustic boundary elements, J. Comput. Acoust. 11(3) (2003) 363-386.

39. P. Langer, M. Güttler, C. Guist, M. Krause and S. Marburg, Six finite elements per wavelength reloaded: The practical use of finite element models and their accuracy in comparison with experimental results, J. Comput. Acoust. 25 (2017) 1750025.

40. H. Wu, W. Jiang, Y. Zhang and W. Lu, A method to compute the radiated sound power based on mapped acoustic radiation modes, J. Acoust. Soc. Am. 135(2) (2014) 679-692.

41. I. Babuska, M. Griebel and J. Pitkaranta, The problem of selecting the shape functions for a p-type finite element, DTIC Document, Tech. Rep., (1988).

42. L. Cremers and K. R. Fyfe, On the use of variable order infinite wave envelope elements for acoustic radiation and scattering, J. Acoust. Soc. Am. 97(4) (1995) 2028-2040. 